\title{
Analysis of Plasma Cyanate as 2-Nitro-5-thiocarbamylbenzoic Acid by High-Performance Liquid Chromatography
}

\author{
Steven Eiger* and Shaun D. Black $\dagger$ \\ Departments of *Physiology and + Biological Chemistry, University of Michigan \\ Medical School, Ann Arbor, Michigan 48109
}

Received September 14, 1984

\begin{abstract}
A method for the determination of cyanate concentration in blood plasma over the range 1 to $1000 \mu \mathrm{M}$ is presented. Cyanate present in the dried residue of acetone-deproteinized plasma is converted to a chromophoric thiocarbamyl derivative by addition of $\mathrm{pH} 3.0$-buffered thionitrobenzoic acid. The derivative is then analyzed by reversed-phase high-performance liquid chromatography with detection at $313 \mathrm{~nm}$, near the absorption maximum. Carbamyl thionitrobenzoic acid peak height is quantified by comparison to a standard curve made by analysis of plasma samples to which known quantities of cyanate have been added. This technique is sensitive and linear with respect to cyanate concentration, and is faster than other reported methods; sample analysis and column regeneration are accomplished within 20 min. (c) 1985 Academic Press, Inc.

KEY WORDS: cyanate; carbamyl thionitrobenzoic acid; high-performance liquid chromatography.
\end{abstract}

It has been speculated that cyanate, a breakdown product of urea, increases in concentration in animals that lack or have malfunctioning kidneys (1). This hypothesis is based on two facts: blood urea levels rise during uremia (2), and cyanate is formed from urea (3). Since cyanate is known to inactivate proteins (4), it has been proposed as a potential uremic toxin. Further, a possible relationship between increased plasma cyanate levels and lowered core temperature has been suggested (5). To investigate these hypotheses it was necessary to measure plasma cyanate levels accurately in uremic animals. Unfortunately, with the exception of an assay reported by Nigen et al. (6), previously published cyanate assays either were not sufficiently sensitive, were not specific for cyanate, or could not be used in plasma or blood (711). The drawback to the assay developed by Nigen et al. was the use of an amino acid analyzer, rendering it costly and slow.

Because the cyanate ion has no absorption in the $u v / v i s$ range, alternative strategies are required to allow its measurement. Therefore, we chose to measure cyanate as a chromophoric derivative formed by reaction with 2nitro-5-thiobenzoic acid (TNB) ${ }^{1}$; cyanate is known to react rapidly with sulfhydryl groups to form an acid-stable thiocarbamyl product $(12,13)$, and this reaction is shown in Scheme 1. The 2-nitro-5-thiocarbamylbenzoic acid (carbamyl-TNB) formed was separated from excess TNB and other constituents in the plasma residue by reversed-phase HPLC, and its absorbance was monitored at $313 \mathrm{~nm}$. Thus, a specific, sensitive, and rapid assay for plasma cyanate has been developed.

\section{MATERIALS AND METHODS}

HPLC-grade $\mathrm{CH}_{3} \mathrm{CN}$ was from Burdick and Jackson, Sequanal-grade trifluoroacetic

\footnotetext{
${ }^{1}$ Abbreviations used: TNB, 2-nitro-5-thiobenzoic acid; DTNB, 5,5'-dithiobis(2-nitrobenzoic acid); carbamylTNB, 2-nitro-5-thiocarbamylbenzoic acid; TFA, trifluoroacetic acid; HFBA, heptafluorobutyric acid.
} 


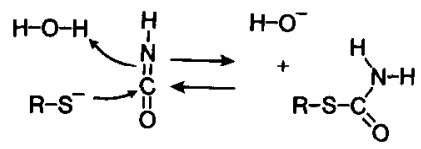

SCHEME 1

acid was from Pierce, heptafluorobutyric acid and tri- $n$-butylphosphine were from Aldrich, and 5,5'-dithiobis(2-nitrobenzoic acid) was from Sigma. All water was glass redistilled and deionized. Sodium cyanate was purchased from Aldrich and recrystallized before use by the technique of Cerami et al. (14). All other chemicals were reagent grade.

Sample preparation. Polypropylene microcentrifuge tubes $(1.5 \mathrm{ml})$ containing heparinized blood were centrifuged at $8000 \mathrm{~g}$ for 2 min in a Brinkman 3200 microfuge. To deproteinize the plasma, $1 \mathrm{ml}$ of acetone was added to $200 \mu \mathrm{l}$ of plasma. This mixture was shaken and centrifuged. The supernatant (1 $\mathrm{ml}$ ) was transferred to a new tube and dried under a stream of nitrogen. Tubes were then capped and frozen until analysis. For construction of standard curves, samples were prepared in the same manner except that known amounts of cyanate were added to distilled water or plasma before deprotcinization.

Assay of cyanate concentration. A stock solution of $2.5 \mathrm{mM}$ DTNB was prepared in $1 / 4(\mathrm{v} / \mathrm{v}) \mathrm{CH}_{3} \mathrm{CN} / 0.2 \mathrm{M}$ citrate-phosphate buffer, $\mathrm{pH} 3.0$, and stored in 1-ml aliquots at $-20^{\circ} \mathrm{C}$. Just prior to use the DTNB was reduced to TNB by addition of $5 \mu 1$ of tri- $n$ butylphosphine (15). Of this TNB reagent $100 \mu \mathrm{l}$ was then added to each deproteinized and dried cyanate sample to be analyzed. After $10 \mathrm{~min}$ of incubation at room temperature $\left(23^{\circ} \mathrm{C}\right)$, the reaction was stopped and the product stabilized by addition of $5 \mu \mathrm{l}$ of $88 \%$ formic acid. Then, an aliquot was analyzed by HPLC with isocratic elution at a flow rate of $1 \mathrm{ml} / \mathrm{min}$, using the following equipment: a Waters RESOLVE spherical C18 column ( $5 \mu \mathrm{m}, 3.9 \mathrm{~mm} \times 15 \mathrm{~cm})$, a Waters liquid chromatograph equipped with a Model 6000A pump, a Model U6K injector (2.0-ml sample loop), and a Model $440 \mathrm{ab}-$ sorbance detector set for operation at 313 $\mathrm{nm}$. After completion of an analysis, the column was regenerated with $4 / 1(\mathrm{v} / \mathrm{v})$ $\mathrm{CH}_{3} \mathrm{CN} /$ running buffer to remove tightly bound compounds such as DTNB, and then reequilibrated with running buffer. Typical analysis and regeneration time was about $20 \mathrm{~min}$.

The effect of $\mathrm{pH}$ on carbamyl-TNB formation was assessed with identical samples of $100 \mu \mathrm{M}$ aqueous cyanate that were submitted to reaction with $5 \mathrm{mM}$ TNB reagent prepared with $0.1 \mathrm{M}$ citrate-phosphate buffers of various $\mathrm{pH}$ values which also contained $25 \% \mathrm{CH}_{3} \mathrm{CN}$. The organic solvent was necessary to enhance the solubility of TNB at low values of $\mathrm{pH}$. Analysis of these samples in duplicate by HPLC was as stated above. Kinetics of carbamyl-TNB formation were assessed by periodic sampling of a reaction mixture which containcd $5 \mathrm{mM}$ TNB and 10 $\mu \mathrm{M}$ cyanate, $\mathrm{pH} 3.0$, at ambient temperature. Aliquots of equal volume from each sample were then analyzed for carbamyl-TNB formation as indicated above.

Standard curves werc gencrated from $A_{313 \mathrm{~nm}}$ peak height of the carbamyl-TNB derivative identified on HPLC tracings, after subtraction of the appropriate blank. These corrected values were plotted against the concentration of added cyanate present in the samples before deproteinization.

Spectrometry. Ultraviolet/visible absorption spectra were recorded at ambient temperature with a Varian Cary 219 scanning spectrophotometer with a $1.0-\mathrm{cm}$ pathlength. Infrared spectra were obtained with a Perkin-Elmer Model 283 infrared spectrophotometer. For these spectra carbamyl-TNB was prepared, purified by HPLC, and analyzed in $\mathrm{KBr}$ pellets. Chemical ionization mass spectroscopy was also performed on HPLC-purified carbamyl-TNB. The mass spectrometer used was a Finnigan 4021 quadropole GC-MS-DS with ionizer temperature of $150^{\circ} \mathrm{C}$, electron energy of $40 \mathrm{eV}$, and methane at 0.3 Torr. 


\section{RESULTS AND DISCUSSION}

\section{Chromatographic Identification of \\ Carbamyl-TNB}

When cyanate in aqueous solution or blood plasma was treated with excess TNB, subsequent HPLC analysis showed the occurrence of a new peak not present in control experiments. This compound, indicated by a star in Fig. 1, was eluted approximately $1 \mathrm{~min}$ before unreacted TNB. The amount of the new compound was dependent on the concentration of both cyanate and TNB, and Gaussian peak shape was maintained with the use of other mobile phases (data not shown). These data are consistent with the identification of the new compound as the adduct of cyanate and TNB, 2-nitro-5-thiocarbamylbenzoic acid. Furthermore, when carbamyl-TNB was collected and rechromatographed, high recovery and identical elution position were observed. Thus, the

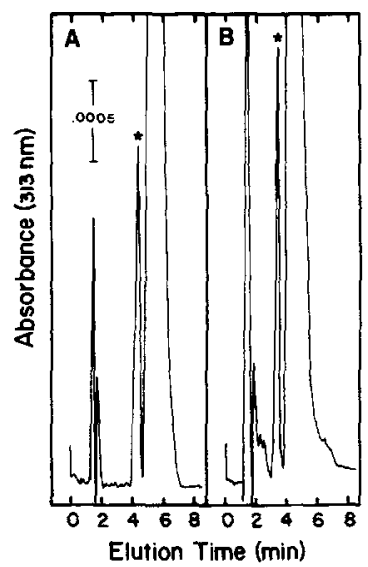

FIG. 1. Reversed-phase HPLC analysis of carbamylTNB. Samples of $50 \mu \mathrm{M}$ cyanate in water (A) or rabbit blood plasma (B) were prepared, derivatized, acidified, and chromatographed as described under Materials and Methods. A $2-\mu$ l aliquot (2\%) of the final sample was injected in each case. The mobile phase used for HPLC analysis of the sample prepared in distilled water (A) was $0.1 \%$ TFA (aqueous), and that used for the plasma sample (B) was $0.1 \%$ HFBA (aqueous). The starred peak in each chromatogram corresponds to carbamyl-TNB, and the large peak following this corresponds to unreacted TNB. apparent quantitative formation, stability, and rapid HPLC analysis of carbamyl-TNB suggested this method as a feasible way to assay cyanate concentration.

Choice of running buffer was crucial at low concentrations of cyanate (i.e., less than $50 \mu \mathrm{M}$ ) when measured in blood plasma since there were substances present that eluted near the carbamyl-TNB peak and absorbed at $313 \mathrm{~nm}$. This necessitated small adjustments either in the percentage of $\mathrm{CH}_{3} \mathrm{CN}$ or in the type of acid in the running buffer. Either mobile phase as used in Fig. 1 should suffice for most applications, although $0.1 \%$ aqueous HFBA is suggested for plasma samples. Up to $10 \% \mathrm{CH}_{3} \mathrm{CN}$ may be necessary to provide a retention volume of approximately $5 \mathrm{ml}$ for carbamyl-TNB on a comparable column. Once a running buffer was selected the elution time was quite stable and varied no more than $1.6 \%$ from run to run.

\section{pH Dependence and Kinetics of Carbamyl- TNB Formalion}

Several solutions of TNB with $\mathrm{pH}$ values in the range 2 to 6 were prepared and these were used to assay identical samples of cyanate. Results of HPLC analyses of carbamylTNB formation for these samples versus $\mathrm{pH}$ showed an approximately Gaussian distribution; the maximal amount of carbamylTNB product was obtained in the region of $\mathrm{pH} \mathrm{3,} \mathrm{with} \mathrm{the} \mathrm{yicld} \mathrm{decreasing} \mathrm{above} \mathrm{and}$ below this $\mathrm{pH}$ optimum. A similar experiment with cyanate added to plasma yielded nearly identical results. Based on these data all assays were done with buffered TNB solutions of $\mathrm{pH}$ 3.0. The decrease in carbamyl-TNB yield below pH 3 was likely due to a decreased concentration of the TNB thiolate anion [thiol, $\mathrm{p} K 4.53$ (16)]. The decrease in yield above $\mathrm{pH} 3$ is likely due to two factors: the decreased concentration of the protonated form of cyanate (i.e., cyanic acid, $\mathrm{p} K$ 2.6) and the increase in the basecatalyzed hydrolysis of the thiocarbamate 
product. In all assays, samples were quenched with formic acid after $10 \mathrm{~min}$ of reaction time. This treatment not only caused a termination of the reaction, but also appeared to stabilize the base-labile carbamyl-TNB product for several hours.

To assess the time dependence of this reaction, reaction mixtures with a low cyanate concentration were quenched at various times and carbamyl-TNB was assayed by HPLC. The results indicated that a maximal formation of product had been reached by $10 \mathrm{~min}$, and that this level was reasonably stable up to $1 \mathrm{~h}$. Based on this, standard reaction conditions were set as $10 \mathrm{~min}$ at room temperature. As noted above, the formic acid quench that is performed after this reaction time serves to stabilize the product formed and thus permits HPLC analyses to be performed without severe time constraints.

\section{Dependence of Carbamyl-TNB Formation on Cyanate Concentration}

Whether cyanate was added to distilled water or blood plasma, the standard curves for carbamyl-TNB formation were linear over a wide concentration range, as shown in Fig. 2. This linearity extended for at least three orders of magnitude, from approximately 1 to $1000 \mu \mathrm{M}$ cyanate. These findings indicate both the efficiency of the chemistry used and the excellent adherence of carbamyl-TNB to Beer's law. Extension of the assay sensitivity to both lower and higher cyanate concentrations should also be possible. In addition, although the plasma data presented were obtained with rabbit plasma, similar results were found when human plasma was used.

Carbamyl-TNB peak height values were slightly higher for the plasma cyanate samples as compared to the aqueous cyanate samples, but the actual respective yields of carbamylTNB were essentially identical. The larger values of peak height for plasma cyanate samples were due to the sharper carbamylTNB peak obtained with the HFBA mobile

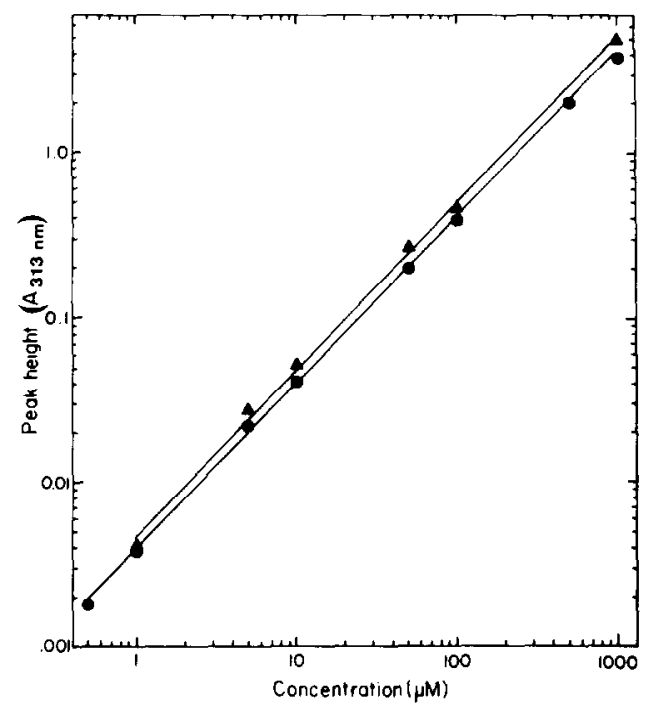

FIG. 2. Dependence of carbamyl-TNB formation on cyanate concentration. Samples of cyanate at various concentrations in distilled water ( $\bullet$ ) or rabbit blood plasma $(\boldsymbol{\Delta})$ were prepared, derivatized with TNB, acidified, and chromatographed as described under Materials and Methods. The mobile phase used for HPLC analysis of aqueous samples was $0.1 \%$ TFA (aq), and that used for plasma samples was $0.1 \%$ HFBA (aq). The results are given as the true $A_{313 \mathrm{~nm}}$ peak height of carbamylTNB; each point represents the average of duplicate determinations. In each case $2 \mu \mathrm{l}(2 \%)$ of the final sample was used for a given HPLC analysis. The SE is within the symbol in each case.

phase; integrated areas between respective plasma and aqueous samples were equivalent. An illustration of this can be seen in Fig. 1 where the peak heights differ by $20 \%$ whereas the integrated areas differ by only $2 \%$. Thus, for routine assays performed with the same chromatographic system, $A_{313 \mathrm{~nm}}$ peak height is quite adequate. However, to compare results obtained with differing mobile phases or columns, integrated areas are required.

\section{Proof of Structure}

Although studics on concentration and $\mathrm{pH}$ dependence indicated the identity of carbamyl-TNB, a rigorous proof of structure was undertaken to firmly establish this assignment. The ir spectrum ( $\mathrm{KBr}$ pellet) of carbamyl-TNB purified by HPLC was consistent 
with the proposed structure: $\nu_{\mathrm{N}-\mathrm{H}}(3450$, $\left.3360 \mathrm{~cm}^{-1}\right), \nu_{\mathrm{O}-\mathrm{H}}\left(3180 \mathrm{~cm}^{-1}, \mathrm{v}\right.$. broad $)$, $\nu_{\mathrm{C}=\mathrm{O}}\left(1700 \mathrm{~cm}^{-1}\right.$, strong, broadened $), \nu_{\mathrm{NO}_{2}}$ (1530, $1350 \mathrm{~cm}^{-1}$, strong), $\nu_{\mathrm{C}-\mathrm{N}}\left(1295 \mathrm{~cm}^{-1}\right)$, $\nu_{\mathrm{C}-\mathrm{s}}\left(715 \mathrm{~cm}^{-1}\right.$, weak). The $1,2,5$ substitution of the phenyl ring was shown by the presence of bands at $870 \mathrm{~cm}^{-1}(\mathrm{w})$ and $830 \mathrm{~cm}^{-1}(\mathrm{~m})$. Further, no free TNB or cyanate was present as evidenced by the absence of absorption in the regions $900-1000$ and $2000-2500 \mathrm{~cm}^{-1}$, respectively. Chemical ionization mass spectrometry showed the $m+1$ ion at $m / e 243$, as expected for carbamyl-TNB. Prominent ions were seen at $m / e 228,200$, and 182 , corresponding to the loss of amine-, amide-, and water from the parent ion.

When carbamyl-TNB was formed at acidic $\mathrm{pH}$ in the presence of excess cyanate, a uv/ visible absorption spectrum with a maximum at $306 \mathrm{~nm}$ was obtained, as shown by the solid spectrum in Fig. 3; this spectrum is also identical to that obtained from HPLC-purified material. A new spectrum, equivalent to that of the TNB-thiolate anion $\left[\lambda_{\max } 410 \mathrm{~nm}\right.$ (16)] was obtained upon raising the $\mathrm{pH}$ to 7.3 , as seen by the dashed spectrum in Fig. 3. When this solution was reacidified, the recorded spectrum did not show a maximum at $306 \mathrm{~nm}$, but rather at $327 \mathrm{~nm}$ (Fig. 3, dotted spectrum), indicative of the protonated TNB thiol (16). These results show that the original compound is highly base labile and readily hydrolyzes to TNB. This finding and the low $\lambda_{\max }$ of $306 \mathrm{~nm}$ in the original spectrum are consistent with the compound being the thiocarbamate of TNB.

Thus, these data prove the structure of 2nitro-5-thiocarbamylbenzoic acid and show the molecular weight to be 242 and $\epsilon_{306} \mathrm{~nm}$ (pH 2.3) to be $6140 \mathrm{M}^{-1} \mathrm{~cm}^{-1}$.

\section{CONCLUSION}

By taking advantage of the reactivity of TNB toward HOCN, and the analytical utility of reversed-phase HPLC, we have developed an efficient and sensitive assay for cyanate.

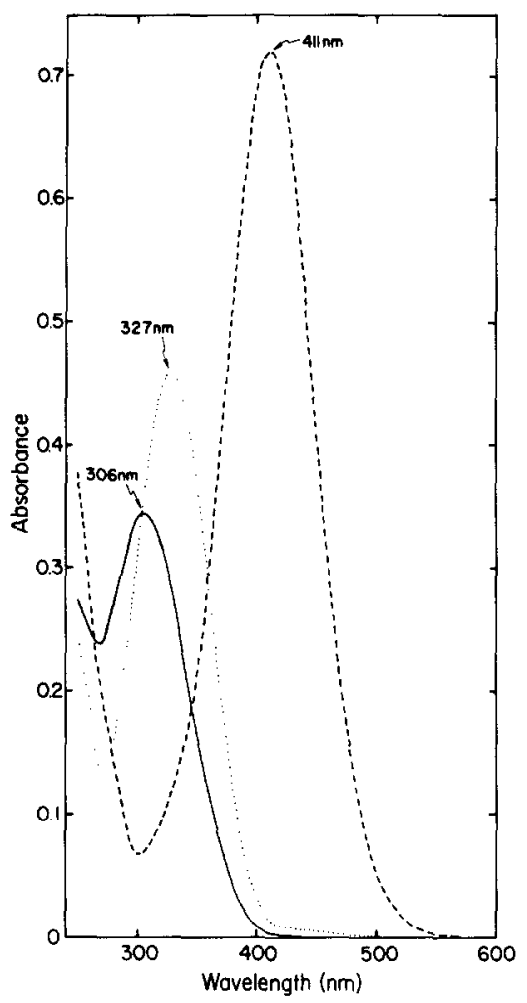

FIG. 3. Spectral evidence for the identity of carbamylTNB. A solution which contained approximately $5 \mathrm{mM}$ TNB, $100 \mathrm{mM}$ cyanate, $\mathrm{pH} 3.0$, was permitted to react at ambient temperature for $10 \mathrm{~min}$ and was then diluted 100-fold with $10 \mathrm{mM} \mathrm{KPO}$, $\mathrm{pH} \mathrm{2.3}$. The solid spectrum (-) was obtained from $1.0 \mathrm{ml}$ of this solution. After addition of $100 \mu \mathrm{l}$ of $1 \mathrm{M} \mathrm{KPO}_{4}, \mathrm{pH} 7.4$, to the cell, the dashed spectrum (---) was recorded. Addition of a small volume of $12 \mathrm{M} \mathrm{HCl}$ to the $\mathrm{pH} 7.4$ solution lowered the $\mathrm{pH}$ to 1.7 , and the dotted spectrum $(\cdots)$ was recorded.

The carbamyl-TNB formed in this reaction is rapidly analyzed by isocratic elution and is quantified by $A_{313 \mathrm{~nm}}$, near the absorption maximum of $306 \mathrm{~nm}$. Further, cyanate has been analyzed in aqueous solution, or rabbit or human blood plasma with equivalent efficiency down to a concentration of at least $1 \mu \mathrm{M}$. The structure of the 2-nitro-5-thiocarbamylbenzoic acid derivative has been proven rigorously.

Relative to existing assays for plasma cyanate, this method requires less expensive equipment and many fewer preparative steps, 
and is more rapid and possibly more sensitive. In addition, if significantly more sensitivity were required, this assay could be modified by substitution of a thiol fluorophore for TNB and the use of fluorometric detection.

Finally, with the use of this assay endogenous cyanate was detected for the first time in both normal and anephric animals and $\operatorname{man}(17)$.

\section{ACKNOWLEDGMENTS}

We thank Dr. G. E. Tarr, Dr. M. J. Kluger, Dr. R. G. Lawton, and Dr. Christin Carter-Su for their encouragement, useful suggestions, and critical reading of the manuscript. This work was supported in part by a grant from The Michigan Kidney Foundation.

\section{REFERENCES}

1. Gilboe, D. D., and Javid, M. J. (1964) Proc. Soc. Exp. Biol. Med. 115, 633-637.

2. Prevost, J. L., and Dumas, J. A. (1821) Ann. Chim. Phys. 23, 90-104.

3. Walker, J., and Hambly, F. J. (1895) J. Chem. Soc. 67, 746 .

4. Carreras, J., Chabas, A., and Diederich, D. (1976) in The Urea Cycle (Grisolia, S., Baguena, R., and Mayor, F., eds.), pp. 501-549, Wiley, New York.
5. Knochel, J. P., and Seldin, D. W. (1976) in The Kidney (Brenner, B. M., and Rector, F. C., eds.), pp. 2137-2183, Saunders, Philadelphia.

6. Nigen, A. M., Peterson, C. M., Gillette, P. N., and Manning, J. M. (1974) J. Lab. Clin. Med. 83, 139-146.

7. Montgomery, E. G. (1971) Biochem. J. 19, 71-74.

8. Dirnhuber, P., and Schutz, F. (1948) Biochem. J. 2, 628-632.

9. Marier, J. R., and Rose, D. (1964) Biochem. J. 7, 304-311.

10. Yates, Y. A., and Grunert, R. R. (1966) J. Infect. Dis. 116, 353-362.

11. Levine, R. L., and Kretchmer, N. (1971) Anal. Biochem. 42, 324-337.

12. Stark, G. R., Stein, W. H., and Moore, S. (1960) J. Biol. Chem. 235, 3177-3181.

13. Stark, G. R. (1964) J. Biol. Chem. 239, 1411-1414.

14. Cerami, A., Allen, T. A., Graziano, J. H., deFuria, F. G., Manning, J. M., and Gillette, P. N. (1973) J. Pharmacol. Exp. Ther. 185, 653-666.

15. Ruegg, U. T., and Rudinger, J. (1977) in Methods in Enzymology (Hirs, C. H. W., and Timasheff, S. N., eds.), Vol. 47, Part E, pp. 111-116, Academic Press, New York.

16. Riddles, P. W., Blakeley, R. L., and Zerner, B. (1983) in Methods in Enzymology (Hirs, C. H. W., and Timasheff, S. N., eds.), Vol. 91, pp. 49-60, Academic Press, New York.

17. Eiger, S. M., and Kluger, M. J. (1984) Fed. Proc. 43,908 . 